\title{
Manejo del drenaje percutáneo guiado por imágenes en un hospital de tercer nivel
}

\author{
Management of imaging guided percutaneous drainage at a third level \\ of care Colombian hospital
}

\author{
Julián Ramírez¹, Yeni Arroyave², Catalina Quilindo', Tania Romero', Carlos Priarone ${ }^{3}$ \\ Médico interno, Facultad de Ciencias de la Salud, Universidad del Cauca, Popayán, Colombia \\ 2 Médica, residente de Cirugía General, Universidad del Cauca, Popayán, Colombia \\ 3 Medico, cirujano general, intervencionismo percutáneo y Cirugía Minimamente Invasiva, Hospital Universitario San José; profesor, \\ Departamento de Cirugía, Universidad del Cauca, Popayán, Colombia
}

\section{Resumen}

Introducción. El drenaje percutáneo de las colecciones intraabdominales, guiado por ultrasonografía o por tomografía computadorizada, ha sido el tratamiento de elección para los abscesos abdominales o pélvicos, entre otros. Objetivo. Describir la experiencia en el manejo percutáneo de colecciones abdominales en pacientes del Hospital Universitario San José de Popayán entre octubre de 2014 y abril de 2016.

Materiales y métodos. Se trata de una serie de casos de 79 pacientes a quienes se les hizo manejo intervencionista de colecciones abdominales por medio de cirugía percutánea guiada por imágenes, los cuales cumplieron con los criterios de inclusión y exclusión. El análisis estadístico se hizo con el programa SPSS Statistics ${ }^{\mathrm{TM}}$.

Resultados. Se realizaron 106 procedimientos percutáneos para drenar diferentes colecciones intraabdominales. La intervención más frecuente fue el drenaje de colecciones hepáticas (32,I \%), en su mayoría, abscesos hepáticos, seguida del drenaje de abscesos posquirúrgicos de cirugías abiertas o laparoscópicas.

Conclusiones. Las técnicas intervencionistas en manos de un cirujano entrenado son un tratamiento eficaz y seguro para las diferentes colecciones intraabdominales. El procedimiento fue exitoso en el $99 \%$ de los pacientes de la presente serie.

Palabras clave: infecciones intraabdominales; absceso abdominal; drenaje; diagnóstico por imagen; ultrasonografía intervencional; tomografía computarizada.

Fecha de recibido: 7/02/2018 - Fecha aceptación: 19/11/2018

Correspondencia: Yeni Arroyave, Departamento de Cirugía, Hospital Universitario San José E.S.E., tercer piso, Popayán, Colombia

Correo electrónico: yarroyave@unicauca.edu.co

Popayán, Colombia

Citar como: Ramírez J, Arroyave Y, Quilindo C, Romero T, Priarone C. Manejo del drenaje percutáneo guiado por imágenes en un hospital de tercer nivel. Rev Colomb Cir. 2019;34:163-70. https://doi.org/10.30944/20117582.110

Este es un artículo de acceso abierto bajo una Licencia Creative Commons - BY-NC-ND https://creativecommons.org/licenses/by-nc-nd/4.0/deed.es 


\begin{abstract}
Introduction: Percutaneous drainage of intraabdominal collections guided by ultrasonography or computerized axial tomography has been the treatment of choice for the management of abdominal or pelvic abscesses.

Objective: To describe the experience with the percutaneous ultrasonography-guided drainage of abdominal collections in patients at the San José University Hospital in Popayán, Colombia, in the period October 20I4 and April 2016.

Materials and methods: This is a case series of 79 patients who underwent interventional management of abdominal collections by a percutaneous procedure guided by images; all patients met the inclusion and exclusion criteria. The statistical analysis was carried out with the SPSS Statistics program.

Results: Io6 percutaneous procedures were performed for drainage of different intra-abdominal collections. The most frequent intervention was the drainage of hepatic collections (32.I\%), mostly hepatic abscesses, followed by drainage of postoperative abscesses following open or laparoscopic surgery.

Conclusions: The management of interventional techniques in the hands of the trained surgeon is an effective and safe method for the treatment of different intra-abdominal collections. The procedure was successful in $99 \%$ of the patients.
\end{abstract}

Key words: intraabdominal infections; abdominal abscess; drainage; diagnostic imaging; ultrasonography, interventional; computed tomography.

\section{Introducción}

El drenaje percutáneo de las colecciones intraabdominales guiado por ultrasonografía (US) o por tomografía computadorizada (TC), ha sido el tratamiento de elección desde hace más de dos décadas ${ }^{1}$. Se considera el tratamiento estándar para el absceso abdominal y el pélvico, en ausencia de indicaciones para la cirugía inmediata ${ }^{2}$, tiene un alto porcentaje de éxito, con baja morbilidad y mortalidad, duración de la hospitalización corta y costos reducidos ${ }^{\mathrm{I}}$.

Según las características de las colecciones, la efectividad del drenaje percutáneo guiado por imágenes puede llegar a estar entre el 80 y el 90 $\%^{3-5}$. La tasa de recurrencia del absceso se estima en 5 a Io $\%{ }^{6}$. La gran ventaja para el paciente es evitarle grandes cirugías con los riesgos que estas conllevan ${ }^{7}$; no obstante, esta técnica no está exenta de complicaciones, como la perforación de algún vaso u órgano (5\%), las infecciones o la muerte (I \%) ${ }^{8}$.

El objetivo de este estudio fue describir la experiencia del manejo percutáneo de colecciones abdominales en pacientes del Hospital Universitario San José de Popayán, para mostrar la eficacia y seguridad del drenaje percutáneo en manos de un cirujano entrenado.

\section{Materiales y métodos}

Se trata de una de serie de casos de pacientes con manejo intervencionista de colecciones abdominales por medio de cirugía percutánea guiada por imágenes en un hospital de tercer nivel de Popayán, Colombia. Se incluyeron los pacientes atendidos en el periodo comprendido entre octubre de 20I4 y abril de 20I6, el cual corresponde a la implementación en la institución del drenaje percutáneo por un cirujano intervencionista y el momento en el que se recolectó la información para el presente estudio.

Los pacientes debían cumplir los siguientes criterios de inclusión: haber sido intervenido quirúrgicamente por colección abdominal mediante cirugía percutánea, ser mayor de 15 años y haber recibido el alta del especialista. Los criterios de exclusión fueron: historia clínica incompleta y falta de observancia de las indicaciones médicas.

Las técnicas usadas fueron la aspiración directa del contenido de la colección sin colocación de catéter y la colocación de un catéter pigtail, 
o doble jota, si estaba indicado, para el drenaje percutáneo de colecciones mayores. Para el drenaje mediante catéter, se empleó la técnica de Seldinger que consiste en la punción mediante aguja seguida de la colocación de una guía en el interior de la aguja, extrayendo después la aguja e introduciendo diversos dilatadores y, posteriormente, el catéter a través de la guía.

Para la recolección de la información, se diseñó un instrumento con las variables de interés, tales como la indicación del procedimiento, el tipo de técnica utilizada para el drenaje percutáneo, la guía imagenológica, el tiempo quirúrgico y las complicaciones del procedimiento, el cual fue diligenciado por los investigadores con la información obtenida mediante la revisión de las historias clínicas.

Las variables continuas se expresaron como la media \pm desviación estándar y, las variables categóricas, como el porcentaje del número total de pacientes analizados o del número total de drenajes realizados.

El análisis estadístico se hizo con el programa SPSS Statistics ${ }^{\mathrm{TM}}$, versión I9 (SPSS Inc., Chicago, IL, USA).

\section{Resultados}

Se incluyeron 79 pacientes en los que se realizaron 106 intervenciones percutáneas para drenar un absceso abdominal, pélvico o abdomino-pélvico, que correspondieron al Ioo \% de los procedimientos practicados en el periodo de estudio. De estos, el $45 \%$ de los pacientes eran de sexo masculino y la media de edad fue de 47,7 años. El 46,8 \% tenía, al menos, una comorbilidad. Las enfermedades concomitantes más frecuentes fueron la insuficiencia cardiaca congestiva, la hipertensión arterial sistémica, la enfermedad pulmonar obstructiva, las neoplasias y la desnutrición. Las características sociodemográficas se presentan en la tabla I.

\section{Procedimientos realizados}

En 79 pacientes, se practicaron Io6 procedimientos percutáneos para drenar diferentes coleccio-
Tabla 1. Características sociodemográficas $(n=79)$

\begin{tabular}{lcc}
\hline Variable & & \\
\hline Edad (media \pm DE) (años) & 47,7 & \\
Grupos etarios (años) & $\mathbf{n}$ & $\%$ \\
$15-20$ & 5 & 6,3 \\
$20-40$ & 22 & 27,8 \\
$40-60$ & 33 & 41,8 \\
$60-80$ & 16 & 20,3 \\
$80-100$ & 3 & 3,8 \\
Sexo & $\mathbf{n}$ & $\%$ \\
Masculino & 45 & 57 \\
Femenino & 34 & 43 \\
Clasificación de ASA* & $\mathbf{n}$ & $\%$ \\
I & 8 & 10,1 \\
II & 36 & 45,6 \\
III & 34 & 43 \\
IV & 1 & 1,3 \\
\hline
\end{tabular}

"ASA: American Society of Anesthesiologists

nes intraabdominales; II pacientes requirieron más de un procedimiento para el drenaje completo, con una mínima y moda de 2 y una máxima de Io procedimientos por paciente. Algunos de los pacientes con complicaciones de pancreatitis aguda grave, habían sido sometidos previamente a una cirugía mayor; estos fueron los que requirieron mayor número de procedimientos para lograr el éxito terapéutico.

La intervención más frecuente fue el drenaje de colecciones hepáticas (32,I \%), en su mayoría, abscesos hepáticos, incluidas las metástasis hepáticas con abscesos; le siguieron el drenaje de abscesos posquirúrgicos de cirugías abiertas o laparoscópicas y el drenaje de colecciones pancreáticas y peripancreáticas por complicaciones de pancreatitis aguda, incluida la necrosis pancreática infectada. En cuarto lugar, estuvieron las colecciones renales y perivesicales, seguidas de los abscesos retroperitoneales por otras causas (tabla 2). Algunos de los pacientes, en especial aquellos con complicaciones derivadas de la pancreatitis aguda, habían tenido manejo quirúrgico abierto previo.

Los pacientes que tenían laparotomías previas para el tratamiento de complicaciones de pancreatitis aguda grave, fueron los que necesitaron mayor número de drenajes percutáneos 
Tabla 2. Procedimientos realizados $(\mathrm{N}=106)$

\begin{tabular}{lcc}
\hline Variable & & \\
\hline Tipo & $\mathbf{n}$ & $\%$ \\
Aspiración & 14 & 13,2 \\
Drenaje & 92 & 86,8 \\
Total & 106 & 100 \\
Diagnóstico & $\mathbf{n}$ & $\%$ \\
Colección hepática-absceso hepático & 34 & 32,1 \\
Colección intraabdominal & 25 & 23,6 \\
posoperatoria & & \\
Colecciones pancreáticas & 22 & 20,8 \\
Colecciones del sistema urinario & 15 & 14,2 \\
Absceso retroperitoneal & 8 & 7,5 \\
Absceso posdiálisis peritoneal & 1 & 0,9 \\
Colecistitis aguda (colecistostomía) & 1 & 0,9 \\
Vía & $\mathbf{n}$ & $\%$ \\
Transperitoneal & 92 & 86,8 \\
Transgástrica & 11 & 10,4 \\
Transglúteo & 3 & 2,8 \\
Técnica & $\mathbf{n}$ & $\%$ \\
Seldinger & 92 & 86,8 \\
Aspiración & 14 & 13,2 \\
Diámetro del catéter (French) (n=92) & $\mathbf{n}$ & $\%$ \\
10 & 34 & 37 \\
8 & 32 & 35 \\
12 & 12 & 13 \\
Otros & 14 & 15 \\
\hline & &
\end{tabular}

para su resolución. La colocación de endoprótesis (stent) doble J transgástrica percutánea, fue una opción terapéutica efectiva en el caso de catéteres transgástricos con drenaje persistente de líquido pancreático, en los casos de fístula pancreática. El lavado continuo con solución salina a través de los catéteres en el caso de la necrosis pancreática infectada, permitió el lavado del lecho pancreático, así como la obtención de fragmentos de necrosis a través de los catéteres (figuras I y 2 ).

El 86,8 \% ( $\mathrm{n}=92)$ de los procedimientos correspondieron a drenaje mediante colocación de catéter y, el I3,2 \%, a aspiración sola sin catéter; todos se hicieron bajo sedación.

Con relación a la técnica, la mayoría de las intervenciones $(86,6 \%)$ fueron procedimientos de tipo Seldinger guiados por ecografía (66\%) y una menor proporción por TC (34\%). Las vías de acceso fueron transperitoneal $(86,6 \%)$, transgástrica (IO,4 \%) y transglútea $(2,8 \%)$, se-

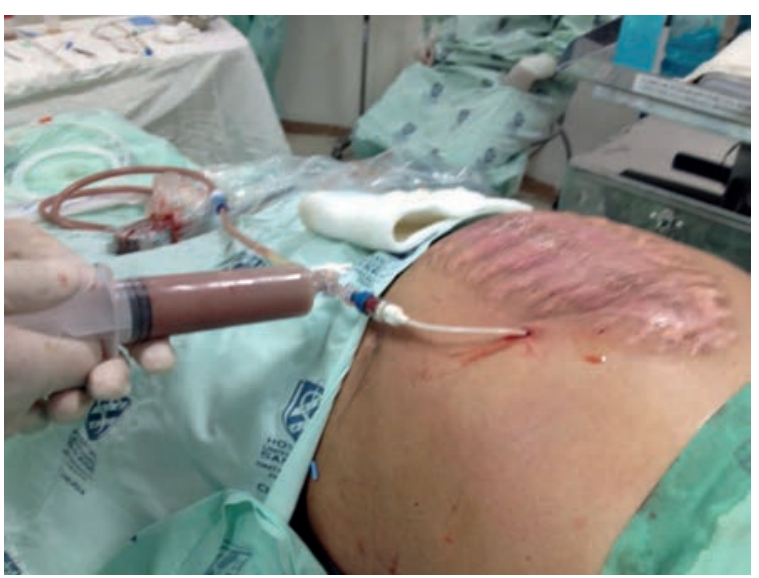

Figura 1. Drenaje guiado por TC de una necrosis pancreática infectada posterior a necrosectomía abierta

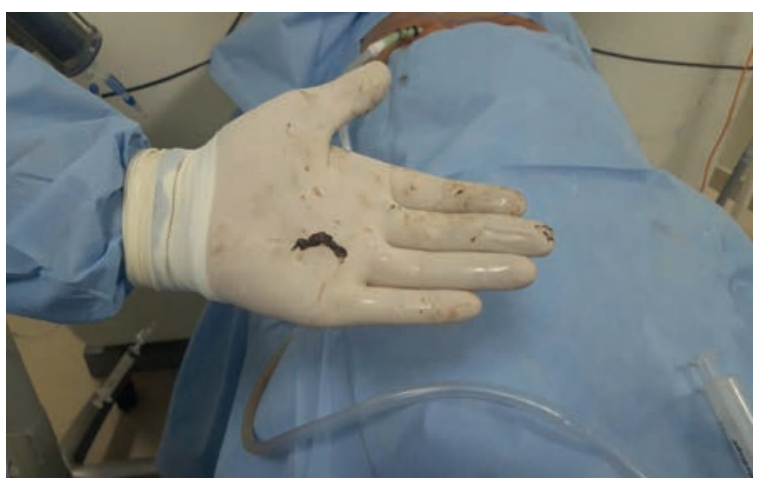

Figura 2. Fragmento de necrosis pancreática infectada

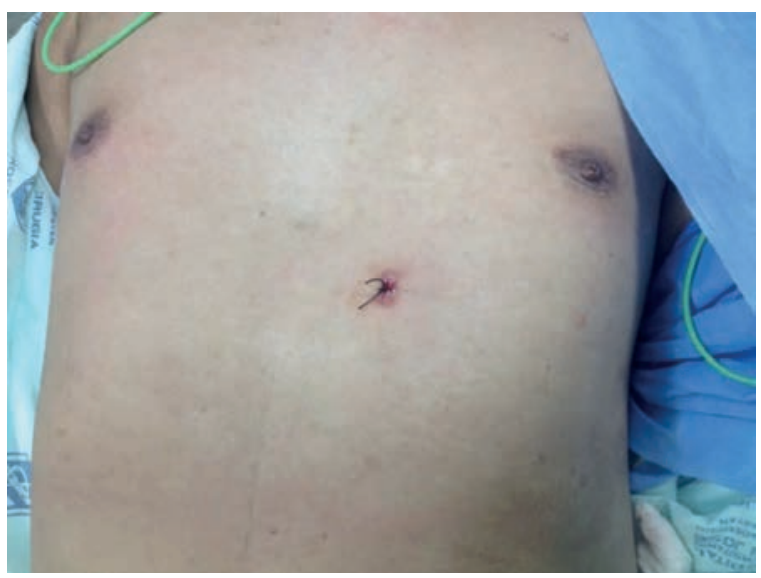

Figura 3. Cicatriz del drenaje transgástrico por necrosis pancreática infectada 


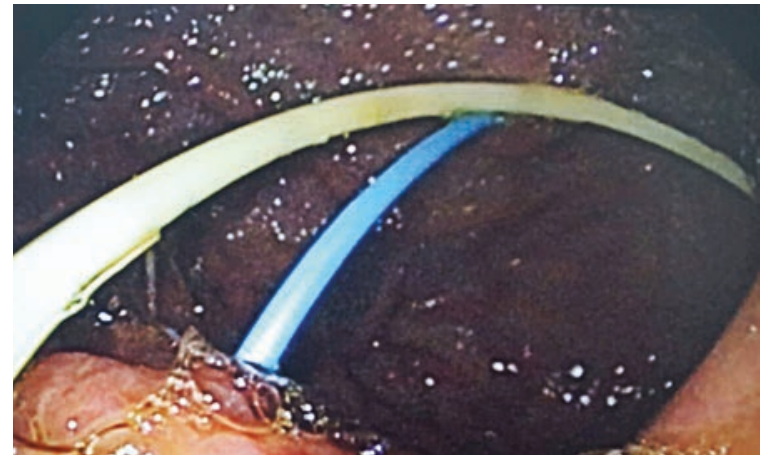

Figura 4. Colocación de catéter doble J transgástrico percutáneo

gún la ubicación de la colección (figuras I-5). La duración media del acto quirúrgico fue de 48,7 minutos (desviación estándar de I9,9 minutos). Los catéteres más utilizados fueron el Io FR y el $8 \mathrm{FR}, 37 \%$ y $35 \%$, respectivamente; y el $64 \%$ de las veces el catéter se mantuvo menos de io días. La mayor parte del material drenado fue de tipo purulento $(64,2 \%)$ con volúmenes que oscilaron entre IO y $2.000 \mathrm{ml}$.

\section{Aislamiento microbiológico}

En 96,2\% (n=IO2) de las intervenciones se hicieron cultivos del líquido drenado, de los cuales el $65,69 \%$ fueron negativos. En los que resultaron positivos, los gérmenes más frecuentemente aislados fueron Escherichia coli, Enterobacter cloacae y Klebsiella pneumoniae (tabla 3).

El tiempo de valoración por cirugía percutánea estuvo en un rango de I a I2O días, con promedio de 22,8, y desviación estándar de 25,2.

El $99 \%(n=105)$ de los pacientes tuvieron un procedimiento exitoso, es decir que se trató la colección mediante drenaje percutáneo, sin necesidad de otro tipo de abordaje. Ninguno falleció.

Solo dos pacientes tuvieron complicaciones asociadas y necesitaron otro tipo de abordaje para su manejo. El primero se retiró agresivamente el catéter para drenar el absceso hepático y presentó hemorragia que se trató mediante laparotomía; el segundo presentó sangrado posterior al drenaje de múltiples quistes gigantes de hígado, el cual fue controlado mediante la embolia vascular selectiva (figura 6).
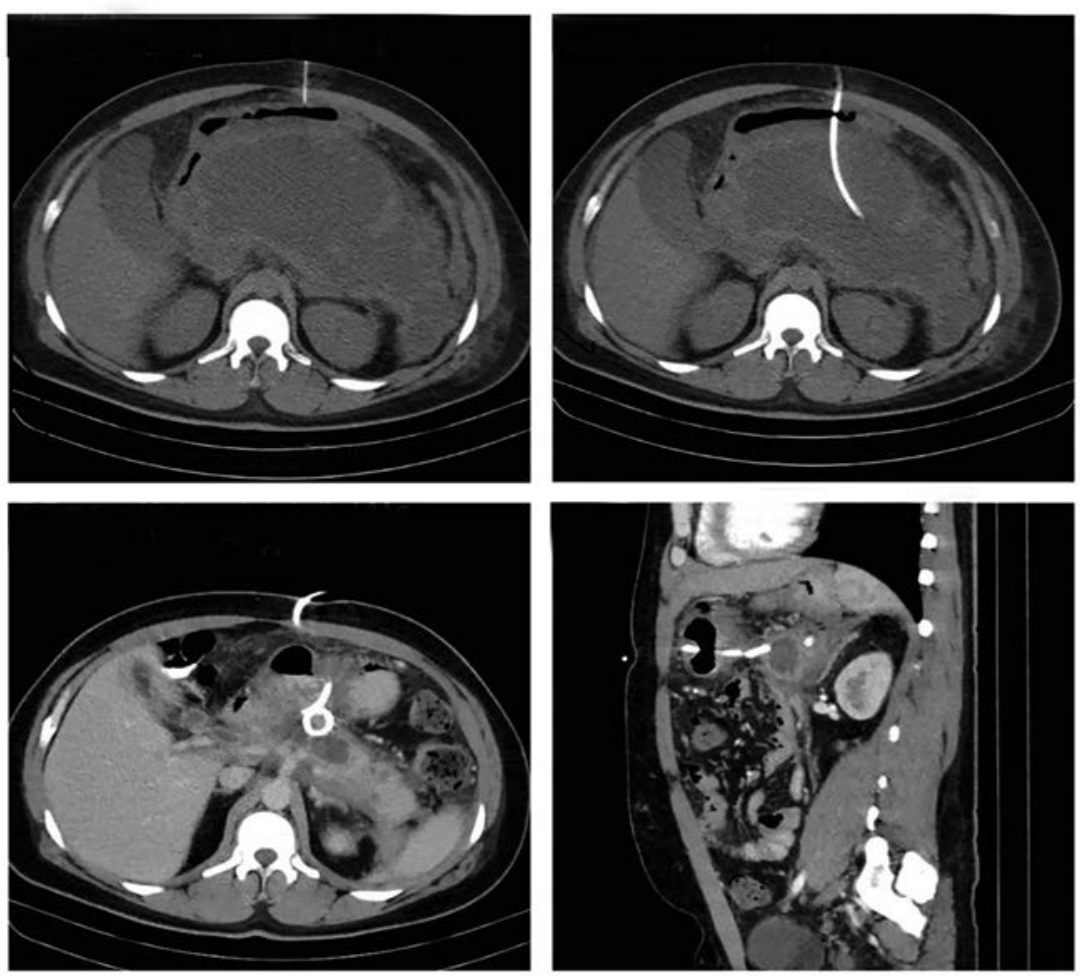

Figura 5. Drenaje percutáneo transgástrico de pseudoquiste pancreático 
Tabla 3. Aislamientos microbiológicos $(\mathrm{N}=106)$

\begin{tabular}{lcc}
\hline Variable & $\mathbf{n}$ & $\%$ \\
\hline Cultivo & 102 & 96,2 \\
Sí & 4 & 3,8 \\
No & 106 & 100 \\
Total & $\mathbf{n}$ & $\%$ \\
Germen aislado & 67 & 65,69 \\
Negativo & 8 & 7,84 \\
Escherichia coli & 7 & 6,86 \\
Enterobacter cloacae & 5 & 4,91 \\
Klebsiella pneumoniae & 4 & 3,92 \\
Enterococcus faecalis & 2 & 1,96 \\
Citrobacter freundii & 2 & 1,96 \\
Proteus mirabilis & 2 & 1,96 \\
Staphylococcus aureus & 1 & 0,98 \\
Candida glabrata & 1 & 0,98 \\
Candida tropicalis & 1 & 0,98 \\
Klebsiella oxytoca & 1 & 0,98 \\
Pseudomonas aeruginosa & 1 & 0,98 \\
multirresistente & 102 & 100 \\
Streptococcus pneumoniae & & \\
Total & & \\
\hline & &
\end{tabular}

\section{Discusión}

El drenaje percutáneo de abscesos intraabdominales ha aumentado constantemente, mientras que el uso del drenaje quirúrgico abierto ha disminuido. Un gran estudio poblacional reveló que más del $80 \%$ de los abscesos abdominales se drenaron por vía percutánea en el 2013.

Con una planificación adecuada previa, el drenaje percutáneo es un enfoque de tratamiento efectivo y seguro, con tasas de éxito superiores al $90 \%$ y menos de ro $\%$ de complicaciones relacionadas en todos los procedimientos, con la ventaja adicional de menor necesidad de anestesia general. Este tratamiento puede ser definitivo o ser previo a una cirugía posterior más planificada y electiva, con menor morbilidad y mortalidad quirúrgicas que si se hiciera de entrada ${ }^{9-13}$.

En el Hospital Universitario San José de Popayán, antes del 20I4, la mayoría de los procedimientos para el drenaje de colecciones intraabdominales eran abiertos. A partir de la implementación del Servicio de Cirugía Per-

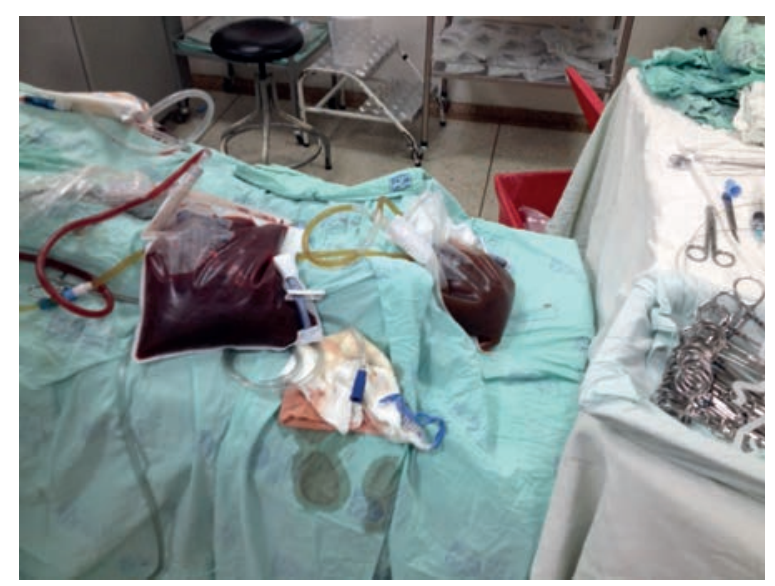

Figura 6. Hemorragia posquirúrgica de quistes hepáticos

cutánea, este enfoque mínimamente invasivo se ha venido incrementando progresivamente. El presente trabajo corrobora la eficacia y la seguridad del drenaje percutáneo guiado por imágenes, con una tasa de éxito del $99 \%$ y una tasa de complicación de I,9\%, tasas incluso superiores a las reportadas en la literatura y mencionadas en el anterior párrafo. Otro tópico a favor de la seguridad del procedimiento, es que el Ioo \% de los drenajes se hicieron bajo sedación y los pacientes no tuvieron que ser sometidos a anestesia general y asistencia respiratoria mecánica.

La técnica más utilizada ( $89 \%$ ) fue la de Seldinger debido a que se realizaron más drenajes que aspiraciones. Por razones de eficacia, la aspiración se utilizó en casos seleccionados, ya que, por ejemplo, en el tratamiento del absceso hepático, cuando se compara la aspiración con el drenaje con catéter, se encuentra que este último es exitoso en el $100 \%$ de los casos y, la aspiración, en el $60 \%{ }^{14}$.

La guía utilizada para los drenajes debe ser en mayor porcentaje ecográfica, para disminuir la exposición a la radiación del paciente y del personal de salud. La guía tomográfica se debe usar en colecciones complejas, que implican una mayor precisión debido a sus relaciones anatómicas y sus posibles complicaciones ${ }^{13}$. 
El método imagenológico más utilizado fue la ecografía, en gran parte, debido al uso fácil y la eficacia de este estudio. Sin embargo, no es despreciable la frecuencia de uso de la guía tomográfica (34\%); esto se debe al gran porcentaje de pacientes incluidos en el estudio con complicaciones de pancreatitis agudas graves, que fueron sometidos a laparotomías previas como parte de su tratamiento, y presentaron abdomen bloqueado y alteración de la anatomía usual.

Aunque el aislamiento microbiológico depende en parte de la etiología y la ubicación del absceso intraabdominal y, por lo tanto, los gérmenes pueden variar entre las diferentes publicaciones, los microorganismos gram negativos son los aislados con mayor frecuencia de las colecciones intraabdominales; E. coli encabeza la lista, tal como se muestra en este trabajo y en otro similar llevado a cabo en Irán. En dicho trabajo ningún agente patógeno creció en casi la mitad de los especímenes; en el presente estudio, el 65,69\% de los cultivos fueron negativos, tal vez porque los pacientes ya habían recibido tratamiento antibiótico antes del drenaje de la colección ${ }^{15}$.

Además de los pacientes con abscesos hepáticos y colecciones posquirúrgicas, aquellos con complicaciones por pancreatitis aguda, incluida la necrosis pancreática infectada, también se beneficiaron del drenaje percutáneo guiado por imágenes. La colocación de la endoprótesis (stent) doble J transgástrica percutánea, fue una opción terapéutica efectiva en el caso de catéteres transgástricos con drenaje persistente de líquido pancreático por fístula pancreática. Esta opción permite el drenaje del líquido pancreático hacia el estómago, sin recidiva de colecciones en el retroperitoneo.

Estos hallazgos, sumados a la baja tasa de complicaciones encontrada, debe motivar al equipo médico y quirúrgico de la institución a usar el enfoque de manejo mínimamente invasivo en casos de complicaciones de pancreatitis aguda, teniendo en cuenta, además, que el enfoque step-up ha sido descrito en la literatura como una estrategia efectiva y segura para el tratamiento de la pancreatitis necrosante infectada; con el drenaje percutáneo, se evita la necesidad de cirugía en la mayoría de los pacientes ${ }^{16}$.

En conclusión, tal como se ha demostrado a nivel mundial, en nuestro medio las técnicas intervencionistas o percutáneas en manos de cirujanos entrenados, son eficaces y seguras para tratar las diferentes colecciones intraabdominales, y la cirugía abierta debería reservarse para casos en los que se contraindique el drenaje percutáneo o este haya sido insatisfactorio.

\section{Referencias}

I. Lucena J. Drenaje percutáneo de las colecciones intraabdominales dirigido por tomografía computarizada. Informe Médico. 2006;8:9I-94.

2. Men S, Akhan O, Köroğlu M. Percutaneous drainage of abdominal abcess. Eur J Radiol. 2002;43:204-I8.

3. Abusedera M, Khalil M, Ali A, Hassan A. Percutaneous image-guided aspiration versus catheter drainage of abdominal and pelvic collections. Egyptian Journal of Radiology and Nuclear Medicine. 2013;44:223-30. doi: Io.IoI6/ejrnm.2013.03.002

4. Cinat ME, Wilson SE, Din AM. Determinants for successful percutaneous image guided drainage of intra abdominal abscess. Arch Surg. 2002;137:845-9.

5. Lorenz J, Thomas JL. Complications of percutaneous fluid drainage. Semin Intervent Radiol. 2006;23:194-204.

6. Gervais DA, Ho CH, O'Neill MJ, Arellano RS, Hahn PF, Mueller PR. Recurrent abdominal and pelvic abscesses: Incidence, results of repeated percutaneous drainage, and underlying causes in 956 drainages. Am J Roentgenol. 2004;182:463-6.

7. Korta I, Del Cura J, Zurera L, Escalante E, González $\mathrm{R}$, Montes $\mathrm{H}$, et al. Drenaje percutáneo de abscesos y colecciones líquidas. Fecha de consulta: 7 de diciembre de 20I7. Disponible en: http://seram.es/readcontents. php?file=webstructure/Io.drenaje_percut\%C3\%Arneo. pdf\&op=download

8. Palavecino P. Ultrasonografía de apoyo en técnicas diagnósticas y terapéuticas. Rev Chil Radiol. 2004;IO:132-8.

9. Levin DC, Eschelman D, Parker L, Rao VM. Trends in use of percutaneous versus open surgical drainage of abdominal abscesses. J Am Coll Radiol. 2015;12(Pt.A):1247-50.

Io. López M, Castro M. Drenaje percutáneo de colecciones abdominales guiados por ecografía. Revista Médica de La Paz. 20II;17:26-30.

II. Rigas DA, Brook OR. Image-guided treatment of abdominal and pelvic abscesses. Semin Roentgenol. 20I7;52:95-IOO. doi: I0.IO53/j.ro.20I6.05.008 
I2. Robert B, Yzet T, Regimbeau JM. Radiologic drainage of post-operative collections and abscesses. J Visc Surg. 20I3;I50(Suppl.):SII-8. doi: IO.IOI6/j.jviscsurg.2013.05.005

I3. Ozer H, Saad WE. Ultrasound-guided abdominal and pelvic abscess drainage. Ultrasound Clinics. 2012:7:347-62.

I4. Bazán S, Pinto J, Godoy D, Campos R, Asmat P, Arias M. Drenaje percutáneo de absceso piógeno hepático: eficacia del manejo. Rev Gastroenterol Peru. 2003;23:17-2I.
I5. Shahnazi M, Khatami A, Jamzad A, Shohitavi S. Safety and efficacy of percutaneous CT-guided drainage in the management of abdominopelvic abscess. Iran J Radiol. 20I4;II:e20876. doi: I0.58I2/iranjradiol.20876

I6. Zerem E, Imamović G, Sušić A, Haračić B. Step-up approach to infected necrotising pancreatitis: A 20year experience of percutaneous drainage in a single centre. Dig Liver Dis. 20II;43:478-83. doi: IO.IOI6/j. dld.2OII.02.02O 